\title{
STEREOTAXIS IN THE TREATMENT OF PARKINSONISM
}

\author{
Leslie Oliver, M.B.(LoÑd.), F.R.C.S., F.A.C.S. \\ Neurosurgeon, Charing Cross Hospital, West London Hospital, and Royal Northern Hospital, London
}

A. M. Hastin Bennett, M.A., M.B.(Camb.), F.R.C.S.

Fellow, Isaac Wolfson Foundation

UNTIL a few years ago the only worth-while operation for Parkinsonism was complete division of the lateral column of the spinal cord at the level of the second cervical segment (Oliver, 1950), but arrest of tremor was obtained at the cost of weakness of the arm and leg and some sensory loss on the opposite side and, furthermore, this operation was applicable to unilateral Parkinsonism only (Oliver, I953). The results of this operation were confirmed by other workers (Sjöqvist, I954; Gaches, 1955). Walker (1949) had tried the effect of dividing the pyramidal fibres in the cerebral peduncle; sensory loss was avoided, but there was considerably greater weakness of the arm and leg than after the cervical operation.

A revolutionary advance in the surgical treatment of Parkinsonism was fortuitously initiated when Cooper (1953, 1954), while exposing the cerebral peduncle to carry out Walker's pedunculotomy, accidentally damaged the anterior choroidal artery and was obliged to coagulate it. (The anterior choroidal artery arises from the internal carotid just above the posterior communicating artery and is adjacent to the cerebral peduncle). The operation was then abandoned, but the patient was nevertheless found to be free from tremor on the opposite side, and none of the anticipated neurological disturbances were produced on this occasion. According to experimental work on the anterior choroidal artery (Abbie, 1933), at least a hemiplegia and homonymous hemianopia were anticipated. As a result of this unexpected experience Cooper continued for a time deliberately to coagulate the anterior choroidal artery for Parkinson's disease. The beneficial effects were attributed to infarction of the globus pallidus as later demonstrated by Mettler, Cooper, Liss, Carpenter and Noback (1954). This operation was, however, soon given up in favour of more certain and less risky methods of attacking the

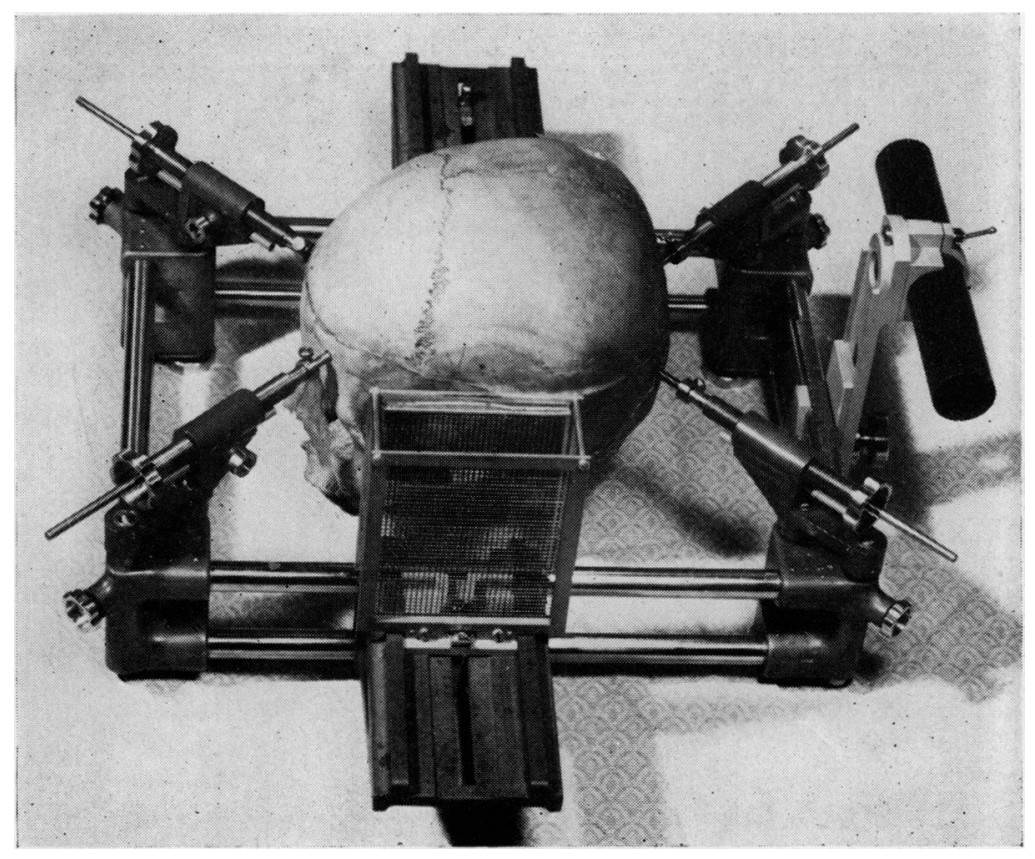

FIG. I.-A lateral view of the stereotaxic frame attached to a skull. The frame is firmly fixed to the bone and also to a specially designed chair (not shown) on which the patient sits. 


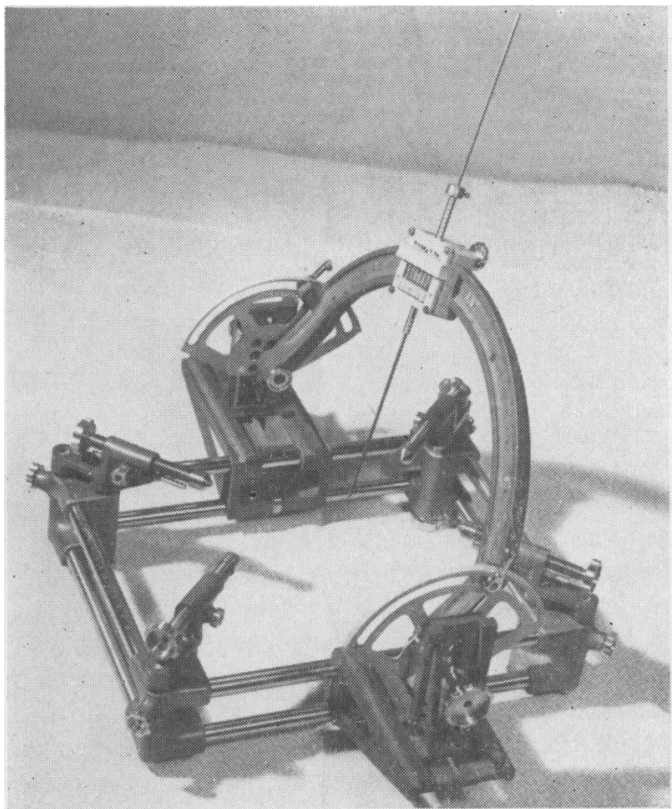

FIG. 2.-The stereotaxic frame with arcuate instrument carrier in position. globus pallidus, such as injection of procaine in oil (Narabayashi and Okuma, 1953) and injection of alcohol (Cooper and Pouloukhine, 1955).

In this work empiricism quickly outstripped scientific knowledge and theory. Thus destruction of cells in the ventral lateral nucleus or the caudal ventral nucleus of the thalamus has been found to be more effective than lesions placed in the globus pallidus (Cooper and Bravo, 1958), although the globus pallidus may still be a worth-while target when lesions placed in the thalamus are inadequate. The operation relieves symptoms on the opposite side. It is carried out on both sides for patients with bilateral symptoms, although at least six months should elapse before treatment of the second side.

There are several ways of locating the nuclei and several ways of inflicting the lesions, but, although there is some variation in the position of the nuclei in relation to the chosen cerebral landmarks, it is exceedingly dangerous to magnify such anatomical variation by use of inaccurate apparatus. Stereotaxic methods which accurately localize the targets in three planes are essential (Spiegel, Wycis, Marks and Lee, 1947; Talairach, Hécaen, David, Monnier and Ajuriaguerra, 1949; Leksell, 1949; Bennett, 1960). Stereotaxis can hardly be better defined than it was by R. H. Clarke in I9I2 in the patent specification of his, the first, stereotaxic apparatus: 'This invention relates to what may be termed stereotaxic surgical apparatus for use in performing operations within the cranium
Fig. 3.-The telescope mounting fixed to the X-ray tube. The telescope is being lined up on the sight seen attached on the right of the frame in Fig. I. By this means the centre ray is made to pass through the double grid on the frame (Fig. I). The chosen landmarks are visualized by positive contrast ventriculography and should appear on the X-ray film in the area where there is no doubling of the grid lines. In this apparatus the X-ray casette is mounted on the side of the frame opposite to that of the grid.

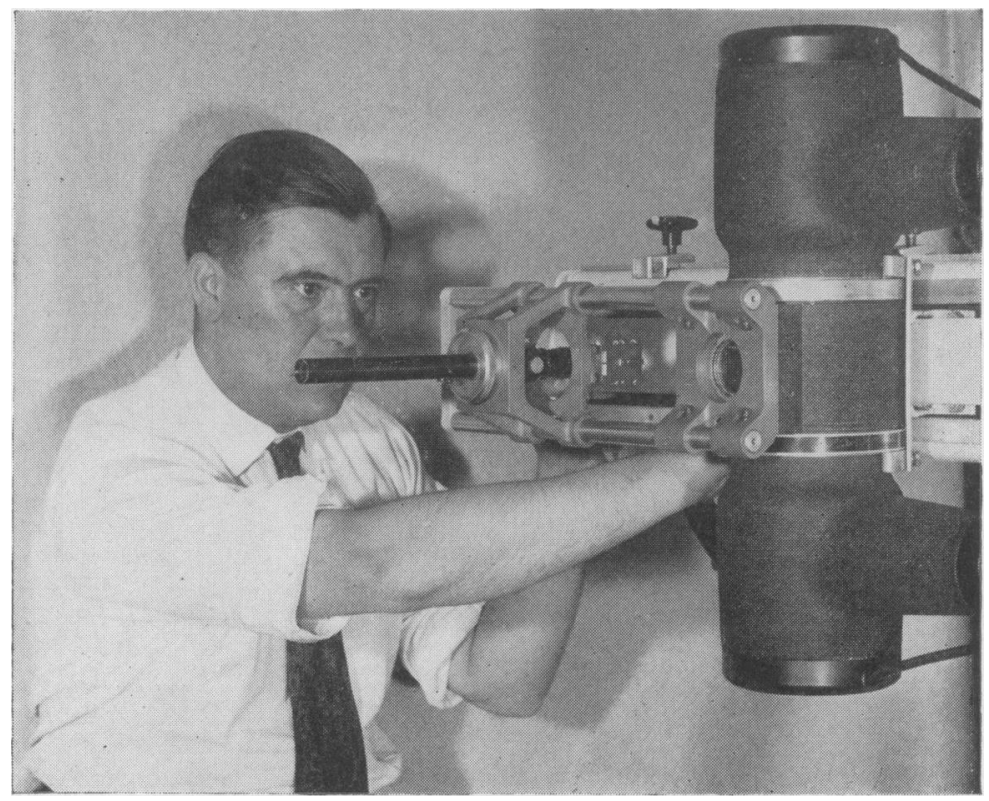




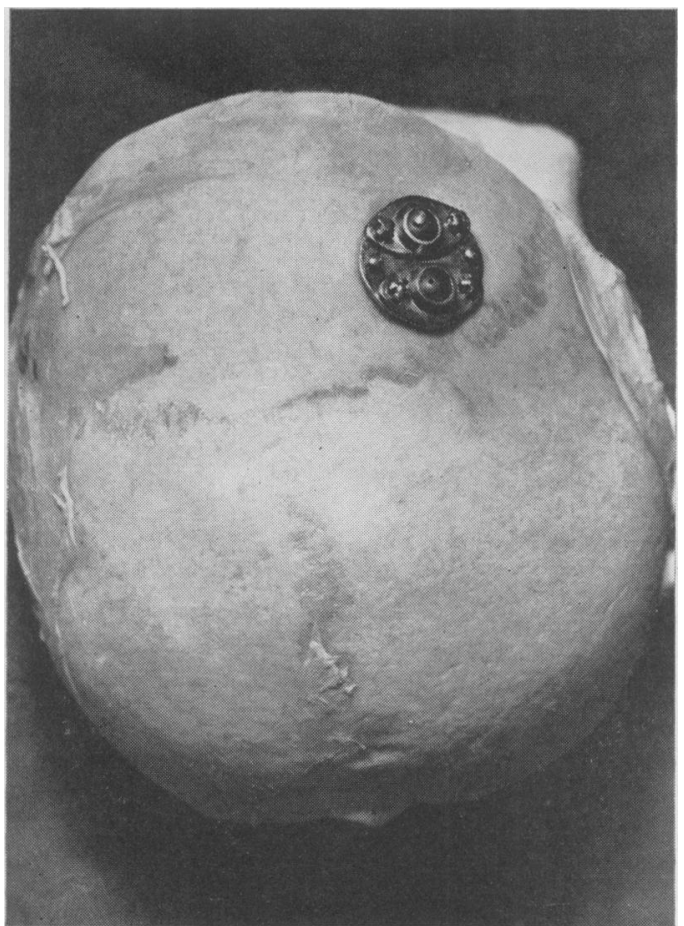

of living human beings ... (it) is designed to enable a so-called probe ... to reach, with absolute precision and by the shortest path, any predetermined point within the cranium through a comparatively small opening formed in the wall of the latter, the primary object being to obviate the necessity of extensively laying open or partially dissecting the head and removing considerable portions of the cranial contents in order to gain access to the exact spot whereat the actual operation is required to be carried out'.

Almost any patients can be treated by the combined use of the Bennett (1960) stereotaxic apparatus (Figs. I, 2 and 3 ) and the modified Oliver (1958) cup and ball (Fig. 4); the former ensures accuracy to I $\mathrm{mm}$. without the necessity for any calculations and the latter allows gradual destruction of the target zones over several days. A detailed description of this technique has been published elsewhere (Bennett, I960). The essential principles are as follows: two immediately adjacent burr-holes are made in the skull on the side opposite that to be treated to accommodate the cup and ball apparatus (Fig. 4). The stereotaxic apparatus is used $(a)$ to place the balls in
Fig. 4.- The double cup and ball apparatus fixed over two burr-holes made in a post-mortem skull. At operation, the anterior ball is set for the ventral lateral nucleus of the thalamus and the posterior ball for the caudal ventral nucleus. If injections through the anterior ball do not have sufficient beneficial effect, injections are given through the posterior ball. Between injections the channels are kept clear by closely fitting obturators. In the illustration the obturator has been removed from the posterior ball.

their cups so that their channels point to the chosen targets, and $(b)$ to measure the depth to which cannulæ have to be passed to reach the targets. Each ball is then firmly fixed in position and the scalp is sutured over the cup and ball apparatus (Fig. 4). On subsequent occasions the stitches are removed under local anæsthesia and injections of a viscid alcoholic mixture (Etopalin $0.2 \mathrm{ml}$.) are given into the appropriate part of the thalamus.

Although some neurosurgeons still regard relief of tremor as the primary aim, restoration of function is the desire of most patients, with relief of tremor of secondary importance (Oliver, 1953). Sometimes oculogyric crises and hyperhidrosis are relieved, but dysarthria and excessive salivation are not. With the use of accurate stereotaxic technique and graduated destruction of the appropriate nuclei, restored or improved function and abolition or reduction of tremor and rigidity can be obtained in most patients of any age with unilateral or bilateral disease, provided there is no associated mental deterioration and they are fit enough to stand a light general anæsthetic for a little over an hour in the sitting position.

\section{REFERENCES}

Abrie, A. A. (1933): The Clinical Significance of the Anterior Choroidal Artery, Brain, 56, 233.

BennetT, A. M. H. (1960): A Stereotaxic Apparatus for Use in Cerebral Surgery, Brit. F. Radiol., $33,343$.

CoOPER, I. S. (1953): Ligation of the Anterior Choroidal Artery for Involuntary Movements-Parkinsonism, Psychiat. Quart., 27, 317. 

COOPER, I. S. (1954): Surgical Occlusion of the Anterior Choroidal Artery in Parkinsonism, Surg. Gynec. Obstet?
99, 207.

f. Amer. Geriat. Soc., 3,839 .

and Bravo, G. (1958): Chemopallidectomy and Chemothalamectomy, $\mathscr{7}$. Neurosurg., 15, 244.

Gaches, J., Le BeAU, J., and TAVERnier, J. B. (1955): Traitement chirurgical des dyskinésies par section complèté du cordon latéral de la moelle cervicale, Sem. Hôp. Paris, 3I, $185^{\circ}$.

Lrksell, L. (1949): A Stereotaxic Apparatus for Intracerebral Surgery, Acta chir. scand., 99, 229.

MetTler, F. A., Cooper, I., Liss, H., CARPENTER, M., and NoBACK, C. (1954): Patterns of Vascular Failure in the Central Nervous System, $\mathcal{F}$. Neuropath., 13, 528.

Narabayashi, H., and OKuma, T. (1953): Procaine Oil Blocking of the Globus Pallidus for the Treatment of Rigidit鼔 and Tremor of Parkinsonism, Proc. jap. Acad., 29, 134.

Oliver, L. C. (1950): Surgery in Parkinson's Disease. Complete Section of the Lateral Column of the Spinal Cord f@्D Tremor, Lancet, $i, 847$.

Oliver, L. C. (1953): 'Parkinson's Disease and its Surgical Treatment.' London: H. K. Lewis. (1958): Cup and Ball Chemopallidectomy Apparatus, Lancet, ii, 401.

SjöQvist, O. (1954): Surgery in Parkinson's Disease, Zbl. Neurochir., 14, 16.

SPIEGEL, E. A., WYCIS, H. T., MARKS, M., and LEE, A. J. (1947): Stereotaxic Apparatus for Operations on the Huma Brain, Science, 106, 349 .

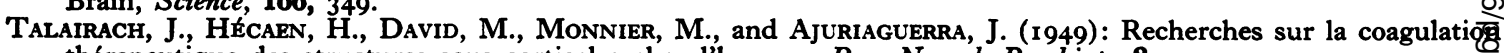
thérapeutique des structures sous-corticales chez l'homme, Rev. Neurol. Psychiat., 81, 4.

WALKER, A. E. (1949): Cerebral Pedunculotomy for the Relief of Involuntary Movements, Acta psychiat. (Kbh.), 24, 723 